altaischen Sprachen zu erbringen gesucht. Demselben Zwecke ist das vorliegende Buch gewidmet, das man ohne ein gewisses Erstaunen nicht lesen kann. Erstaunen muss man nämlich darüber, dass nach der ganzen Entwickelung der Etruskologie und besonders, seitdem Pauli die wïste Art der Forschung, die auf diesem Gebiete eingerissen war, durch seinen scherzhaften Versuch, das Etruskische als eine den baltischen Sprachen nahe verwandte darzustellen, gründlich beleuchtet hat, noch ein derartiges Buch überhaupt auf dem Markte zu erscheinen wagt. Das beste an dem Buche ist die Einleitung, welche eine kleine Uebersicht teils über die Auffindung des etruskischen Sprachmaterials teils über die Geschichte der Etruskologie gibt. Sobald der Verfasser dann in die eigentliche Untersuchung eintritt, wird sofort der Boden einer gesunden Sprachforschung verlassen. Zunächst sucht er eine Art Verwandtschaft zwischen den arischen und den altaischen Sprachen zu konstruieren, nicht zwar eine eigentliche Verwandtschaft, aber doch eine Verwandtschaft, die $\delta v \nu \alpha ́ \mu \varepsilon \iota$ für beide Sprachgruppen von vornherein vorhanden war; diese zeigt sich besonders in den Endungen und in den Wurzeln. Dem hierauf begründeten Nachweis, dass das Etruskische teils mit der einen teils mit der anderen der beiden Gruppen übereinstimmt, folgt dann eine besondere $\mathrm{Be}$ handlung von Götter- und anderen Namen. Damit schliesst der erste Teil, im zweiten Teil werden "les documents étrusques" behandelt, und zwar in fünf Kapiteln, in denen der Verfasser sich um die gesicherten Resultate der bisherigen Forschung so wenig wie möglich kümmert. Mit der vom Verfasser befolgten Methode ist ungefähr die Verwandtschaft jedes etruskischen Wortes mit jedem beliebigen Worte irgendeiner altaischen oder indogermanischen Sprache erweisbar. Ein paar Beispiele dürften zur Charakterisierung dieser Methode genügen; auf S. 76 heisst es wörtlich: "Aphrodite, $\bar{K}$ ypris, Cybèle, Cupra, Hécube et Niobé, sont des mots de la racine $K u b$ (KAB), indiquant l'idée de multiplication, de gonflement et de maternité. La palatale initiale $K$ est tombée dans Aphrodite (V. Rac. ab); elle est précédée d'une voyelle dans Hécabe; elle est nasalisée dans Niobé. En étrusque, on a une forme de Kypris qui est Akhufitr; $a$ est préfixé, l'r est déplacés. Auf S. 80 lesen wir: "Clytemnestre, variante de Clytumne et de Clytie, mot de la racine alt, galt (V. TAL) signifiant brillant, or; cf. gold. Les noms de la famille des Tyndarides renferment tous cette idée de beauté et d'éclat, et coincident avec les noms des métaux; celui de Tyndare lui-même avec le nom du zinc".
Es wird nichts anderes möglich sein, als über das Buch zur Tagesordnung überzugehen.

S. Eitrem: Beiträge zur griechischen Religionsgeschichte, I. Der vor-dorische Widdergott (Christiania VidenskabsSelskabs Forhandlinger for 1910 Nr. 4). Christiania, Jacob Dybwad, 1910. $24 \mathrm{~S} .8^{\circ}$. Besprochen ron C. Fries, Berlin.

Der Verfasser geht von dem Gotte Karnos aus, den die Dorier bei ihrem Eindringen in Lakonien vorfanden, und hält ihn für einen Widdergott. Man wird vielleicht im Hinblick auf das Semitische und auf lateinisches cornu, deutsches: Horn letztere Bedeutung: Horn für die ursprüngliche halten. Das würde an sich mit Eitrems Deutung: Widder harmonieren. Nun hat Ernst Siecke (Götterattribute $228 \mathrm{ff}$.) gezeigt, wie das Horn als göttliches Attribut auf die Mondnatur des betreffenden Dämons schliessen lässt. Siecke bemerkt: „Die Mondsichel wurde gern mit einem Horn oder mit einem Doppelhorn verglichen. Hörner bei einer göttlichen Gestalt pflegen daher ein sicheres Kennzeichen ihres Mondursprunges zu sein", und auch Apollon wird gelegentlich mit Hörnern dargestellt (l. c. 229). Vielleicht ist auch der Karneios im Grunde nichts als der altachaiische Mondgott, dessen Kult die Dorier übernahmen. Eitrem verfolgt die Bedeutung des Widders in der Mythologie und erblickt in ibm das Sinnbild der Liebe und Fruchtbarkeit. Siecke dagegen erkennt im Widder eine Tierform des Mondes (1. c. 248). Der goldene Widder des Phrixos, der beim Fall der Helle zugleich ein Horn verlor, ist ihm ein lunares Wesen (1. c. 229), wohl nicht mit Unrecht. Hermes, den Eitrem für einen Widdergott bält (S. 5), ist dies nach Siecke (Hermes der Mondgott 84) vermöge seiner lunaren Natur. Der Widder ist dem Hermes "in den mannigfachsten Verbindungen zugesellt" (1. c. 84). Im folgenden werden dann die Widdergottheiten einzeln behandelt und dann gibt Verfasser eine detaillierte Darstellung der Karneenfeier. $\mathrm{Ob}$ die Kombinationen Eitrems im einzelnen sich bewähren werden, steht dahin; dass er den Orient ausser acht lässt, ist ein bedauerlicher Mangel; im übrigen ist nicht zu leugnen, dass Eitrem auch bier Gediegenes und Gründliches geboten hat, wenn die Ausbeute neuer überzeugender Gedanken auch nicht allzugross ist. Die neuere Literatur ist gut benutzt und sorgfältig zitiert.

Sprechsaal.
Encore un mot à propos de VR 47 r 30-31.
Par François Martin.
Peiser reprend dans OLZ, octobre 1911, col. 477-479, ma traduction de ce passage. Son-interprétation de hur ša an paraît très 
plausible. Pour ib-bir-ru, il a raison aussi, mais en partie seulement.

Il est évident que $i b$-bir-ru n'est pas un nifal. Je me suis aperçu de ce lapsus quand j'avais déjà envoyé la plupart des exemplaires du tirage à part, et je n'ai pu faire de correction manuscrite que sur un petit nombre d'entre eux.

Mais cette forme n'est pas davantage la $1^{\text {èr }}$ pers. sing du parfait qal de 'br, comme le croit Peiser: s'il en était ainsi, nous aurions $i(c)$-bir-ru et non $i b$-bir-ru ; de plus, la finale $u$ n'aurait guère de raison d'être dans une proposition non subordonnée, dont le verbe, à $3^{\circ}$ radicale forte, serait au singulier. A la fin de la même phrase, à la 1 . 32, où les verbes sont certainement à la $1^{\text {ère }}$ pers. du singulier, ils n'ont pas de voyelle finale: am-ma-rit .....a appa-tar. Peiser a si bien senti la difficulté q'il supplée wo: „dem Platze des Rechts der Wenschen (wo) ich gebannt war", - ou "(wo) ich hinüberging".

$i b$-bir-ru, arec redoublement de la $2^{\circ}$ radicale et $u$ final, est donc l'imparfait qal de ebêru, à la $3^{\mathrm{e}}$ pers. du pluriel.

Ce verbe n'est pas ebêru "passer de l'autre côté". Dans le Code de Hammourabi, il ne sert jamais d'équivalent ì alâku ou à šalù; s'il se trouve dans leur voisinage, c'est uniquement parce qu'il exprime une des phases du drame qui se terminait par la marche vers le fleuve et l'immersion dans le fleuve. Voir en particulier les passages tout $\dot{a}$ fait convaincants à cet égard: Code, V 27, a-vii-lum a-wi-lam ú-ubbi-ir et V 2, $70 m u-z u$ i $u-u b-b i r-s i$.

C'est le verbe ebêru "lier", qui a ce sens même. au qal: voir Brünnow, No. 7517 et 7533 où le signe $\hat{x-}=$ ebêru et șabâtu.

Il semble done qu'il faut traduire VR 47, r 30 a-šâr di-en nišê ib-bir-ru: „là où on rend (litt. lie) le jugenent des hommes".

Zum Vorstehenden bemerke ich, dass ich freilich irrtümlich mit dem Aorist übersetzt habe, während ibbiru präsens ist, wie ippušu; also wird besser zu fassen sein: "dem Platze des Rechtes von Menschen, die hinübergehen". F. E. P.

\section{Altertumsberichte. Museen.}

Von den Neuerwerbungen der Berliner Museen in den Monaten August bis November 1911 seien erwähnt: Aegyptische Abteilung: Gipsabguss einer Wasseruhr in Kairo aus der Zeit Amenophis III. Stück einer Prunkpalette mit Darstellung von Giraffen und Steinböcken aus ältester Zeit (um 3500 v. Chr.). Bronzener Türbeschlag mit dem Namen Darius I. Bronzene Türangel mit dem Namen des Amerdis (um 700 v. Chr.). Kalksteinrelief des neuen Reiches; Darstellung eines Kriegswagens. Griechischer Grabstein, der Tote trägt den nubischen Eigen- namen Milanja. Bruchstücke eines kampanischen Terra. kottareliefs in römisch-ägyptischem Mischstile. Schale mit Kranzornament aus bluuer Fayence. - Vorderasiatische Abteilung: Eine altbabyl. Hymnentafel. Ein Abguss eines in Konstantinopel befindlichen aramäischen Reliefs aus Sendschirli. - Islamische Kunstabteilung: Gegossene Silberschale mit figürlichen Darstellungen in Relief, ursprünglich vergoldet; Persien, frühe Sassanıdenzeit. Vier kleine, frühislamische Glasgefässe mit gegossenen Verzierungen.

(Amtl. Ber. a. d. Kgl. Kunstsamml., Okt. 1911 bis Jan. 1912).

Wdr.

Bei der Aufschüttung eines Schutzwalls zur Verteidigung der Oase von Ain-Zaira haben die Italiener jüngst ein sebr gut erhaltene Mosaik aus römischer Zeit an den Tag gefördert. Sch.

\section{Aus gelehrten Gesellschaften.}

In der Sitzung der Académie des Inscriptions et Belles-Lettres rom 15. Dezember 1911 berichtet Holleaux über eine wichtige griechische Inschrift, die aus den letzten Grabungen von Delos stammt. Sie stellt die griechische Uebersetzung eines römischen Senatsbeschlusses dar, wurde nach Delos durch atbenische Strategen gebracht und bezieht sich auf den Kult des Serapis. Holleaux möchte die Inschrift etwa ins Jahr 165 v. u. Z. setzen.

In der Sitzung der Société des Antiquaires de France vom 6. Dezember 1911 berichtet H. de Villefosse über zwei Inschriften, die Merlin in Südtunis entdeckt hat, und teilt die Entdeckung zweier prächtiger Marmorsarkophage durch P. Delattre in Karthago mit.

(Chronique des Arts, 23. Dec. 1911.)

$\mathrm{Wd} \mathbf{r}$.

\section{Mitteilungen.}

Die University of Pennsylvania und Hilprecht. Wir wir dem Public Ledger (Philadelphia) rom 12. Dez. 1911 entnehmen, hat die sich an den Rücktritt Bilprechts anschliessende Kontroverse nunmehr durch die Entscheidung der dortigen Universitätsbehörden ihr Ende gefanden. Damit erübrigt sich ein weiteres Eingehen auf die unerquickliche Angelegenheit. Da aber, veranlasst durch Bilprechts Anhänger Radau, eine Anzahl europäischer Gelehrter sich bei der Universität Philadelphia um eine Wiederanstellung Hilprechts in Philadelphia bemüht haben, und da durch die hierbei erfolgten Bemühungen gewiss manchem nur eine einseitige Darstellung des Konflikts zur Kenntnis gekommen sein dürfte, so teilen wir den an Herrn Prof. Zimmern gerichteten Schlussbrief des Provosts of the University in extense mit: Dear Sir - The trustees of the University of Penasylvania have had under careful consideration your communication written in August last. By their direction and on their behalf, I now submit a formal reply.

You and your associates, we feel sure, are sensible of the delicacy of the position in which you placed yourselves by addressing such a letter to the University of Pennsylvania. Yon assumed, without inquiring of us what the facts of the case really were, that our officials had deliberately violated Doctor Hilprecht's private rights and had been guilty of the wanton destruction of University property.

You will permit us to question the propriety of making preliminary assumptions such as these. Courteous as were the terms in which your communication was couched, it was hard for us to overlook what, in the case of less eminent correspondents, we should have resented as a grave breach of academic comity.

In view, however, of your distinguished attainments, and of the services which you and your assiociater have rendered to the cause of sound learning, the trustees 